\title{
El consentimiento informado y la autonomía del paciente en Cuba. Un binomio indispensable
}

\author{
Recibido: 2 de junio de 2020 • Aprobado: 4 de octubre de 2020 \\ https://doi.org/10.22395/ojum.v20n42a13
}

\author{
Liuver Camilo Momblanc \\ Universidad de Oriente, Santiago de Cuba, Cuba \\ liuverc@uo.edu.cu, liuvercamilo@nauta.cu \\ https://orcid.org/0000-0002-1311-095X \\ Juan Carlos Mendoza Pérez \\ Universidad de Guantánamo, Guantánamo, Cuba \\ juanc1989@nauta.cu, jcperez@cug.co.cu \\ https://orcid.org/0000-0003-3197-9605
}

\section{RESUMEN}

Este trabajo está motivado por la innegable problemática que genera la deficiente regulación del consentimiento informado y la autonomía de la voluntad de los pacientes en el ordenamiento jurídico cubano, unido a su insuficiente conocimiento y desarrollo en la práctica médica. En este sentido, a partir del análisis de los presupuestos teóricos y prácticos de esta institución, y el estudio de derecho comparado, se fundamentan propuestas para su perfeccionamiento. Para la siguiente investigación fueron utilizados como métodos generales de las investigaciones científicas: el analítico-sintético y el inductivo-deductivo, además de los específicos de las investigaciones jurídicas: exegético-jurídico y jurídico-comparado. Estos métodos están apoyados por la técnica investigativa de la revisión bibliográfica. Como principal resultado, se ofrece una propuesta de resolución al Ministerio de Salud Pública dirigida a la adecuada regulación del consentimiento informado, la autonomía de la voluntad de los pacientes y el derecho de información sanitaria, sobre la base de los presupuestos teóricoprácticos sistematizados en la investigación, que constituyen la guía de orientación en el proceso de su interpretación y aplicación.

Palabras clave: consentimiento Informado; autonomía del paciente; presupuestos teórico-prácticos; regulación jurídica. 


\section{Informed Consent and Patient Autonomy in Cuba: An Essential Binomial}

\section{ABSTRACT}

This work is motivated by the undeniable problem generated by the deficient regulation of informed consent and autonomy of will of the patients in the Cuban judicial ordinance, linked to insufficient knowledge and development in the medical practice. Thus, based on the analysis of the theoretical and practical presuppositions of this institution and a study through comparative law, the study proposes some improvements. This research employed the analytic-synthetic and the inductive-deductive methods, as well as those proper to the judicial researches: the judicial-exegetic and the judicialcomparative ones. These methods are supported by the bibliographical review research technique. As the main result, this study proposes a resolution to the Public Health Ministry, directed towards the adequate regulation of the informed consent, the autonomy of the patients will, and the right to sanitary information, based upon the theoretical and practical presuppositions systematized in the research and that constitute guidelines in the process of its interpretation and application.

Keywords: informed consent; patient autonomy; theoretical-practical presuppositions; legal regulation. 


\section{INTRODUCCIÓN}

Este artículo es resultado de la actividad investigativa de sus autores y corresponde a la línea de investigación del Departamento de Derecho de la Universidad de Guantánamo. Para esta investigación se tuvo en cuenta: i) el consentimiento informado, la autonomía del paciente y la vinculación de estos con el derecho; y ii) el capítulo de derecho penal de la Unión de Juristas de Cuba en Guantánamo.

La medicina cubana goza de gran prestigio y reconocimiento nacional e internacional. Ello obedece a la admirable labor que sus galenos realizan con altruismo y los diversos avances que obtiene el país en esta rama (Danel Ruas, 2020; Morales Ojeda et al., 2018; Redacción Minsap, 2019). Los médicos cubanos, con pocos recursos pero con mucha preparación y humanismo, son capaces de salvar vidas en cualquier parte del mundo. Estos son solo algunos de los elementos que sustentan la confianza de la población cubana hacia los profesionales de este sector y el predominio de un modelo de marcado tinte paternalista.

Lo anterior se traduce en una primacía del principio de beneficencia sobre el de autonomía. Culturalmente la población cubana tiende a acatar las opiniones de los galenos y estos disfrutan de la libertad de ejecutar los actos que parezcan necesarios en beneficio del paciente (Arrue Caraballo y Azaharez Leal, 2012, p. 50; Risco Turiño et al., 2016).

Pero en un contexto global en el que la relación médico-paciente es uno de los aspectos que en el ámbito médico destaca por su variación a lo largo de la historia - y en los últimos años - mediante su tránsito de un esquema a otro (Lifshitz, 2015, p. 151; Patiño Restrepo, 2012, p. 97; Santos Echaide, 2016, p. 3); en el que se pregona con mucha fuerza un modelo de autonomía respetuoso de la autodeterminación del paciente; es notaria la trascendencia que se le ha concedido al intitulado consentimiento informado como mecanismo legitimador del acto médico. En este sentido, como apunta Gómez (2003):

No es de extrañar que desde las coordenadas de una ordenación estatal que proclama como uno de sus pilares básicos la autonomía y el derecho al libre desarrollo de la personalidad del individuo, suela reconocerse de forma prácticamente unánime que el tratamiento médico reclama, como presupuesto mínimo de su conformidad a derecho, la concurrencia del consentimiento del paciente. Solo cuando este asiente en acudir a la consulta, en revelar sus datos íntimos y en someterse a una terapia, la mayoría de las veces invasiva de su integridad física, el acto médico puede adjetivarse como legitimado. (p. 35)

Por ello, es imprescindible la realización de un estudio sobre esta institución médico-jurídica en Cuba que permita la evaluación de la regulación normativa y la formulación de propuestas encaminadas a su efectivo desarrollo, tratándose de un tema que nos involucra a todos como beneficiarios de los servicios de salud. 
Esto se ha vuelto especialmente claro en el mundo editorial, donde se constata el creciente interés que existe sobre este tema, como lo ilustra el abundante patrimonio bibliográfico que lo rodea. Entre las obras destacan libros, tesis (de grado, maestría y doctorales) y artículos científicos, escritos desde diferentes enfoques y esferas de la actuación médica y jurídica en diversos países. Todos de importante validez orientadora para la elaboración del marco conceptual teórico de la investigación, en relación con sus principales coordenadas. No obstante, como se refieren a la regulación y aplicación del consentimiento informado en sus respectivos países, en su mayoría están influenciados por análisis locales que pueden diferir de nuestra cultura y costumbre. Este es el caso de los trabajos de Agón López (2016); Alfaro Carballido y García Rupaya (2011); Cadenas Osuna (2018); Loring Martínez de Irujo (2013); Novoa Reyes (2013); Ochoa Ulloa (2017); Peña Machuca (2017); y Pérez Dávila (2017).

En el escenario nacional, aunque con un menor caudal, también existen trabajos que desde diferentes perspectivas estudian el consentimiento informado. Pero, en general, adolecen de propuestas concretas para su adecuada concepción, esclarecimiento y puesta en práctica en el país. Asimismo, por lo general, abordan el objeto de estudio desde la perspectiva particular de la ciencia médica y sus diferentes especialidades (pediatría, cardiología, anestesiología, entre otras). Igualmente, omiten requisitos y características esenciales de esta institución. No obstante, destacan los trabajos realizados por González López et al. (2009); Núñez de Villavicencio Porro (2006); Risco Turiño et al. (2016); Valenzuela y González (2010); y Vila y Pérez (2013).

En esta dirección, también se estima insuficiente el reconocimiento jurídico del consentimiento informado en el país si se tiene en cuenta que la Ley 41 (1983) de Salud Pública no regula de forma expresa esta institución; en tanto otras disposiciones del Ministerio de Salud Pública solo se refieren sucintamente a ella. Por consiguiente, se aprecian lagunas que generan inseguridad e incertidumbre en el tráfico sociojurídico asociado al desarrollo de esta institución legitimadora del acto médico. También, como lógica consecuencia, ello puede incidir negativamente en la actividad judicial y administrativa de solución de conflictos en torno a la responsabilidad jurídica del médico frente a posibles reclamos de pacientes y familiares. De igual forma, su inadecuada implementación puede dejar, en ciertos casos, en estado de indefensión a los profesionales de la salud ante la ocurrencia de un resultado adverso del cual haya podido informar previamente al paciente.

Tales manifestaciones motivan la realización de una indagación sobre el consentimiento informado y la autonomía de los pacientes en Cuba que permita identificar las deficiencias presentes en su reconocimiento jurídico y promover la necesidad de su perfeccionamiento legislativo. Además, tiene gran significación social, pues constituye, para la sociedad cubana, la garantía del expreso reconocimiento normativo del derecho a la autonomía de la voluntad en el marco de la relación entre el médico y 
el paciente. Como aspecto relevante, se destaca que el estudio no se circunscribe a ninguna de las especialidades de la medicina en particular, sino que se abordan las coordenadas generales del consentimiento informado. En este sentido, se puede aprovechar como base bibliográfica fundamental, en función de posteriores incursiones que se quieran realizar en especificidades de la ciencia médica. Lo más importante es que, a diferencia de sus precedentes investigativos, se realizan propuestas para lograr una correcta normativización de esta institución en el país. De esta manera, con la presente exploración científica se espera obtener los siguientes resultados: un diagnóstico de las deficiencias del consentimiento informado en Cuba; material bibliográfico actualizado que pueda ser consultado por estudiantes y profesores de las especialidades médica y jurídica; y significativas propuestas normativas que sirvan de bases para la elaboración de una resolución del consentimiento informado en Cuba.

\section{ANÁLISIS JURÍDICO-DOCTRINAL DEL CONSENTIMIENTO INFORMADO}

Para lograr una correcta aplicación del consentimiento informado como institución médico-jurídica es ineludible incursionar en aquellos elementos que lo definen y caracterizan. Con este objetivo se considera necesario analizar su evolución histórica y conceptualización; además, sus principales elementos: sujetos, objeto, forma y naturaleza jurídica. Las próximas cuartillas se dedican, sin pretensiones filosóficas, al análisis de estos aspectos.

La medicina es una de las profesiones más antiguas de la humanidad cuya razón esencial es la curación de los enfermos. Sin embargo, "la historia del consentimiento informado, considerando la delimitación conceptual del término realizada por diversos autores, se puede considerar como reciente, pues se inicia a partir de la mitad del siglo xx" (Bernar Borda, 2000, p. 16). En los inicios de la medicina se evidenciaba un modelo paternalista de la relación médico-paciente que se fortaleció con el juramento hipocrático, en ese momento no era posible hablar de la exigencia del consentimiento informado.

Las primeras noticias de esta institución se encuentran en la jurisprudencia norteamericana con los siempre citados casos: Mohr vs. Williams (1905) y Schloendorf vs. Society of New York Hospital (1914) (Agón López, 2016; Bandrés et al., 2010; Bernar Borda, 2000; Casa Madrid Mata, 2004, p. 20; González Fernández et al., 2012; Mellado, 2016, p. 345). No obstante, algunos autores plantean que "las primeras sentencias y discusiones sobre la necesidad de aceptar los procedimientos y de informar a los pacientes [...] no comenzaron en EEUU, como se suele pensar, sino en Inglaterra y Francia en los siglos xviII y xix" (Bandrés et al., 2010, p. 18). En esta misma dirección también se destaca Loring Martínez de Irujo (2013).

En cualquier caso, estos primeros fallos judiciales en los que se reconocía como expresión de su autonomía — en el marco de las actuaciones médicas— el derecho de 
los pacientes a consentir o no las mismas, sentaron las bases de una jurisprudencia de gran influjo en la solución de los sucesivos procesos sobre el tema. No obstante, existe cierto acuerdo en la literatura científica al considerar que fue en el caso Salgo vs. Leland Stanford Jr. University of Trustees (1957) (citado en, Bernar Borda, 2000, p. 25; Restrepo et al., 2012) — sentencia del Tribunal Supremo del Estado de California— donde se acuñó el término consentimiento informado como expresión del respeto del derecho de los pacientes a su autonomía. Ello se traduce en el correlativo deber de los facultativos de ofrecer la información acerca de los procedimientos médicos a los que van a ser sometidos los pacientes, para que estos puedan decidir libre y voluntariamente si lo consienten (Bernar Borda, 2000, p. 25; Restrepo et al., 2012).

En el orden propiamente normativo, el Código de Núremberg (1947) y la Declaración de Helsinki (1964), se refirieron a la necesidad de contar con el consentimiento del sujeto, aunque esencialmente en el contexto de las investigaciones o experimentos en seres humanos. Además, Vila y Pérez (2013) afirman que la primera carta magna en consagrar el derecho a la autonomía de la voluntad en la relación médico-paciente y, por tanto, el consentimiento informado, fue la Constitución italiana (1947). En ella se estableció que: "Nadie puede ser obligado a un determinado tratamiento sanitario si no es por disposición de la Ley. La ley no puede en ningún caso violar los límites impuestos por el respeto a la persona humana" (Vila y Pérez, 2013, p. 136).

Otros de los hitos normativos, lo establece la Carta de los Derechos del Paciente (1973) aprobada por la Asamblea de los delegados de la American Hospital Association. En este instrumento se "reconoce que el paciente tiene el derecho a recibir del médico la información necesaria para dar un consentimiento informado antes de someterse a cualquier tratamiento" (Bernar Borda, 2000, p. 35).

También tiene este carácter la Constitución de España (1978), base de la Ley General de Sanidad (1986)' que dio paso a la actual Ley 41 (2002) de Salud en ese país. De igual modo, como señalan Vila y Pérez (2013), marca una pauta el Convenio de Oviedo (1997). Entre sus disposiciones este convenio establece que: "Una intervención en el ámbito de la sanidad solo podrá efectuarse después de que la persona afectada haya dado su libre e inequívoco consentimiento" (Vila y Pérez, 2013, p. 136).

En cuanto a la definición del consentimiento informado, han sido tantos los autores e instituciones que no serían suficientes estas cuartillas para sistematizar una muestra representativa de ellas. Sin embargo, de su examen, a pesar de los diferentes

Esta, tratándose ya de una ley específica en materia de salud, consagró entre los derechos de los pacientes, el de consentir sobre el tratamiento que recibirían del médico al exigir "que se les dé en términos comprensibles, a él y a sus familiares o allegados, información completa y continuada, verbal y escrita, sobre su proceso, incluyendo diagnóstico, pronóstico y alternativas de tratamiento. A la libre elección entre las opciones que le presente el responsable médico de su caso, siendo preciso el previo consentimiento escrito del usuario para la realización de cualquier intervención" (Vila Morales y Pérez Echeverría, 2013, p. 136). 
enfoques, no se aprecian diferencias sustanciales. Todas las definiciones giran en torno a los elementos característicos o invariantes de lo que actualmente llamamos consentimiento informado. Estos elementos son, como sostiene Bernar Borda (2000), "la información al paciente y el derecho del mismo a tomar parte en las decisiones que le afectan" (p. 24).

Lo anterior es independiente de que algunos lo consideren como un acto jurídico, y lo ubiquen dentro de un marco contractual entre el médico y sus pacientes, donde se reflejan obligaciones para ambas partes (Vila y Pérez, 2013). En este sentido, otros lo consideran como el resultado de un mero iter de la actuación médica en el que los profesionales de la salud aportan una gran información de la enfermedad, para luego esperar que sus pacientes acepten el tratamiento correspondiente (Sánchez Arrastía y Contreras Olivé, 2014). Incluso, hay quienes lo conciben como un proceso gradual y verbal (González Fernández et al., 2012).

No obstante, en relación con su naturaleza jurídica, corresponde al pensamiento común que el consentimiento informado está basado sobre un contrato especial de confianza entre el paciente y el médico. Así lo certifican los autores que resaltan el elemento del consentimiento como acuerdo de voluntades que implica querer lo mismo y cuyo estudio corresponde a la teoría general del contrato (Bernar Borda, 2000; Chepe Ramos, 2010; Vila y Pérez, 2013)

Como resultado del escudriñamiento de las diversas definiciones, se considera que el consentimiento informado es aquel proceso que surge en el marco de la relación médico-paciente, se manifiesta de forma verbal o escrita y constituye un acto jurídico de responsabilidad bilateral. Mediante este los médicos informan a sus pacientes, capaces jurídicamente, o, en su defecto, a sus familiares sobre su estado de salud, aportándoles el diagnóstico de su enfermedad, los riesgos y beneficios que pueden tener y otras alternativas al respecto para que luego, en pleno uso de su derecho de autonomía, decidan libremente, sin coacción o amenaza, si se someten o no al tratamiento médico. Para comprender el consentimiento informado como institución jurídica, es necesario analizar, desde esta perspectiva, sus principales elementos constitutivos: subjetivos, objetivo y la forma.

En primer lugar, el elemento subjetivo se refiere a los sujetos que intervienen en tal proceso y que jurídicamente se entienden como sujetos activo y pasivo. Por un lado, el sujeto activo es el paciente, quien de una forma u otra dirige la relación, pues de él depende si se continúa o no el procedimiento médico correspondiente. Por otro lado, el sujeto pasivo es el facultativo, quien deberá informar al paciente y esperar a que este decida autónoma y libremente sobre el tratamiento a efectuar.

También hay autores que consideran como un sujeto principal en el proceso, además de los citados, a la administración sanitaria (Vila y Pérez, 2013). No obstante, 
es más acertado considerarlos como sujetos secundarios, de conjunto con el personal sanitario que interviene en el tratamiento del enfermo y los testigos instrumentales (presentes en el hecho solo para darle veracidad a la actuación realizada en ese momento), porque estos sujetos con su participación no definen las invariantes esenciales del consentimiento.

Por otra parte, al hablar de los sujetos se debe hacer referencia a la capacidad de los pacientes. De ella depende no solo la validez del consentimiento, sino su clasificación en consentimiento directo o por representación (Agón López, 2016; Ochoa Ulloa, 2017; Pérez Dávila, 2017). En esta dirección, ante la innegable incapacidad del paciente para decidir y el otorgamiento de esta institución, tiene relevancia la intervención de una tercera persona legitimada a tales efectos (los tutores legales). En ausencia de cualquiera de estas personas sustitutas, se estima justificada la intervención del médico, quien deberá actuar basado en la ética profesional y en el principio de beneficencia (Valenzuela y González, 2010p. 12).

Una mayor complejidad reviste cuando el enfermo tiene su capacidad de decidir restringida, porque en tales casos no se le excluye del procedimiento de información sanitaria, sino que se ha de tener en cuenta su criterio sin decidir directamente sobre ello. Por lo tanto, en tales procederes no se prescinde de la representación legal para otorgar el consentimiento, ya que el paciente no ostenta la capacidad plena para tomar decisiones propias. El problema en esta situación es que se debe valorar el modo en que el profesional médico debe informarle al paciente, de manera tal que este pueda comprender la realidad de su enfermedad. Para ello es necesario el apoyo de los familiares y especialistas en psicología, y otras materias, en función de que el enfermo no se altere psicológicamente y entienda la información ofrecida (Valenzuela y González, 2010, p. 12).

En segundo lugar, otro elemento constitutivo del consentimiento informado es el objeto, que comprende su contenido. Valenzuela y González (2010) afirman que el objeto de esta institución es el tratamiento médico por realizar en el paciente. Sin embargo, se considera factible comprender como tal la información ofrecida por el médico a sus pacientes y consecuentemente la aceptación o no de estos sobre la realización del tratamiento correspondiente. Esa información comprende los riesgos, beneficios, medios con los que se pueden lograr y los fines por los que se pueden alcanzar estos.

Finalmente, en tercer lugar, el elemento formal no se refiere solamente a la formalidad que se requiere para su constitución, sino que entiende, además, su forma de realización: directo o por representación (Vizcaya Benavides et al., 2014). En relación con la formalidad como elemento estructural del consentimiento informado alude a la forma en la que exterioriza la manifestación de la voluntad autónoma de los pacientes. Sobre este tema se estima conveniente que se utilice la forma escrita, no 
como una institución con carácter ad solemnitatem, que se realiza por la mera solemnidad del proceso; sino que es ad probationem, que posee carácter de prueba fehaciente en determinados procesos (Valenzuela y González, 2010).

Los autores consideran que la constancia probatoria de esta institución se debe obtener a través de la realización de un documento firmado por ambos sujetos, que será insertado en la historia clínica del paciente (Menacho Ángeles, 2018). No obstante, también puede ser verbal, pues en determinados diagnósticos no se considera necesario la forma escrita. Este es el caso cuando el tratamiento sanitario no es tan riguroso o invasivo para el enfermo ni supone riesgos o inconvenientes de notoria y previsible repercusión negativa sobre su salud. En tales casos, se exige que se utilice siempre la forma expresa de la voluntad del paciente. La constancia de esta forma verbal y expresa de la institución, se piensa que sea la confección de un sencillo documento en el que el médico expone que se tuvo en cuenta el consentimiento informado del paciente antes de iniciar el procedimiento sanitario, y que de igual forma se anexará a su historia clínica.

El consentimiento informado, tanto en su forma escrita como verbal, puede realizarse de manera directa o por representación. Es directo cuando se efectúa por los propios sujetos principales (activo y pasivo) que intervienen en el proceso. Mientras que el consentimiento por representación se aplica cuando existe algún problema con la capacidad de los pacientes (por ejemplo, menores de edad e incapacitados mentalmente). Pero el mayor problema se encuentra cuando se refiere a personas que, de forma inmediata, se han determinado incapaces para decidir y no se han establecido sus representantes legales.

\section{CARACTERISTIICAS, REQUISITOS, FASES Y LÍMITES DEL CONSENTIMIENTO INFORMADO}

El consentimiento informado es un concepto relativamente nuevo que traduce un derecho del paciente dentro de la bioética médica, y su principal objetivo es proteger la autonomía de este. Sin embargo, su aceptación en el marco de la relación médicopaciente no está exenta de tensiones. De ahí surge la necesidad del dominio de las principales coordenadas de esta categoría como herramienta teórica que tribute a la correcta identificación e implementación de esta institución. En esta tesitura se asumen y sistematizan, entre otros, los atributos que según Vila y Pérez (2013) lo distinguen.

Garantiza el derecho de autonomía de los pacientes: exige que los profesionales de la medicina respeten íntegramente el derecho que tiene la persona de poder decidir ante las diversas situaciones que se puedan presentar durante el proceso de atención médica.

Restringe y garantiza un derecho para el facultativo: al fundamentarse en la autonomía del paciente, limita al facultativo para efectuar el tratamiento médico si así lo ha querido el mismo. Además, lo protege respecto a los eventos adversos que deriven 
del proceder de la medicina no imputables, pues el paciente conoció previamente sobre los posibles riesgos que luego aceptó y asumió.

Es de aplicación general: en una ciencia médica cada vez más fragmentada, no es exclusivo de ninguna de sus especialidades. Sus principios básicos y presupuestos bioéticos se aplican a todas las esferas de la práctica médica.

Obliga al facultativo a brindarle información al paciente: para que el tratamiento se concrete los médicos deben informar detalladamente del proceder en sí, de sus riesgos y beneficios, y brindar alternativas de tratamiento.

La exteriorización de la voluntad debe ser siempre expresa: significa que la manifestación de voluntad debe constar por escrito una vez leído y firmado por las partes el documento correspondiente (en casos necesarios se requerirá la presencia de testigos). Ahora, cuando el proceder sea sencillo puede ser verbal, esto es, en casos en los que el tratamiento sanitario no es tan riguroso o invasivo para el enfermo, ni supone riesgos o inconvenientes de notoria y previsible repercusión negativa sobre su salud (Ochoa Ulloa, 2017; Sanz Rubiales et al., 2016; Seuba Torreblanca y Ramos González, 2003).

Compromete al médico con el paciente en todo el proceso asistencial: los obliga a realizar la atención médica como está previsto en la práctica médica, y a atender todas las complicaciones que deriven de la intervención siempre que esté a su alcance y competencia profesional.

Genera responsabilidad para el paciente: el paciente queda responsabilizado por ocultar conscientemente información médica necesaria para la efectividad del tratamiento, a la cual el médico no tiene acceso. En consecuencia, si por causas de información ocultadas por el paciente se obtienen resultados infaustos, dado el caso, los galenos se encuentran libres de responsabilidad, si actuaron de buena fe.

Convergencia interdisciplinar: se enmarca dentro de las ciencias médicas y jurídicas en tanto se desarrolla en el ámbito de las relaciones médico-paciente. Consiguientemente, constituye un elemento legal pues su deber es proteger un derecho. En este sentido, genera consecuencias de esa naturaleza para ambas partes en caso de incumplimiento.

Responsabilidad dual: la responsabilidad, una vez concertado el consentimiento informado, no solo puede ser del profesional médico que atiende al paciente, también puede evaluarse la de la entidad administrativa en la que este se desempeña.

Carácter individual: supone que se tenga un protocolo de actuación médica para el consentimiento informado por cada enfermedad y especialidad de esta ciencia y que, además, se individualice según las características de cada paciente. Ello se explica por 
el hecho de que no todos los enfermos tienen la misma capacidad bilógica, psicológica o las condiciones socioambientales para asimilar procederes médicos idénticos.

Elevación de la calidad de los servicios médicos: posibilita que la práctica médica se contemple en un ambiente sano y autónomo para los enfermos. También mejora los atributos de legalidad de la historia clínica como instrumento médico jurídico en los casos en que se requiera. En este sentido como sostiene Chepe Ramos (2010):

Cuanto mejor sea la relación en términos de respeto mutuo, conocimiento, confianza, valores compartidos y perspectivas sobre las enfermedades y la vida, mejor será la cantidad y calidad de la información sobre la enfermedad del paciente que se intercambiará en ambas direcciones, mejorando la precisión del diagnóstico y aumentando el conocimiento del paciente sobre la enfermedad o dolencia. (p. 9)

Una vez expuestas las características, es importante tener en cuenta los requisitos, pues sin su observación es posible poner en duda la validez o efectividad del consentimiento informado. En el patrimonio bibliográfico se observaron discrepancias entre los autores en torno al tema. Algunos, como Vila y Pérez (2013) se refieren a cuatro requisitos: la necesidad del consentimiento, la comunicación adecuada, la capacidad legal de las partes y la objetividad de la intervención. Otros, como Lugones Botell et al (2005) mencionan: la libertad de decisión, la validez y autenticidad de la información, y la ausencia de control externo. Sin embargo, la mayoría de los autores, concuerdan en que no deben faltar para la aplicabilidad de la institución: la voluntariedad, la capacidad para decidir, la información suficiente y la comprensión de la información.

En primer lugar, la voluntariedad se refiere a que el paciente debe consentir sobre el tratamiento correspondiente a su enfermedad, de forma autónoma y libre de todo tipo de vicio. Significa que en la decisión del paciente no debe existir manipulación, persuasión o coacción alguna que perjudique su voluntad (Vera Carrasco, 2016).

En segundo lugar, la capacidad para decidir se fundamenta en que el paciente debe, en principio, estar capacitado para comprender la situación real de su enfermedad y ser capaz de tomar decisiones al respecto. No obstante, se pueden dar casos en los que no ostente la plena capacidad de decidir propiamente, cuestión que proporciona la necesidad de contemplar y aplicar el consentimiento por representación (Vila y Pérez, 2013).

En tercer lugar, también se exige que la información otorgada por el médico sea suficiente, entendida como la necesaria e imprescindible para la toma de decisión por el enfermo. Para ello, el profesional médico debe informar a sus pacientes en términos comprensibles. El enfermo no necesita saber todo cuanto existe de su enfermedad, pues los elementos técnicos sanitarios los puede dominar solo el profesional. La información que se le debe aportar solo requiere contener la naturaleza de su enfermedad, los objetivos del procedimiento a realizar (diagnóstico o terapéutico), los riesgos y 
efectos secundarios que pudieran darse, las alternativas y beneficios esperados (Vera Carrasco, 2016).

Como último requisito, y estrechamente relacionado con el anterior, está la necesidad de que el sujeto activo haya comprendido eficazmente la información ofrecida por el médico. La necesidad de comprensión de esta se desarrolla en dos elementos importantes: la objetiva y la subjetiva. Según Vera Carrasco (2016) la primera se refiere a la comprensión de todo lo relacionado con la enfermedad y el tratamiento correspondiente. Esta contiene elementos muy técnicos de la profesión, por lo que el médico debe hacer uso de una terminología adecuada que posibilite esa comprensión. Y la subjetiva se manifiesta en la capacidad del paciente para entender la realidad sanitaria, donde interviene la representación legal del mismo para otorgar ese consentimiento (consentimiento por representación).

El proceso en el que se aplica el consentimiento informado consta de dos fases: una en la que interviene el profesional de la salud y la otra en la que interactúa el enfermo o su representante legal, en caso de consentimiento por representación. En la primera fase, el médico debe informar a sus pacientes y familiares, si así lo desean, sobre su enfermedad y tratamiento, con la calidad y cantidad necesaria para comprender la realidad a resolver. Y en su segunda fase, el paciente, una vez informado y con la comprensión suficiente sobre el tratamiento a realizar, decide, como un ser totalmente autónomo, si está de acuerdo o no con que se inicie el procedimiento sanitario correspondiente.

El objetivo principal del consentimiento informado es que el paciente consienta autónoma y libremente sobre la realización de un tratamiento médico, correspondiente a su estado de salud. Ello no se puede cumplir sin la previa información que al respecto el médico debe proporcionarle al mismo. Esta razón permite afirmar la complejidad de la difícil tarea de determinar cuál de estas fases constituye la más importante en este proceso. No obstante, podemos señalar que la primera tendría mayor connotación que la segunda, pues constituye el medio para el final otorgamiento de este consentimiento. Esto significa que de la forma en la que se desempeñe la fase informativa depende la aceptación del paciente. En este sentido, si la información otorgada es errónea, o se encuentra viciada por manipulación, persuasión o coacción, entonces el consentimiento no se considera válido a los efectos médico-jurídicos.

De esta manera, al mismo tiempo que la institución del consentimiento informado se fundamenta en el derecho de autonomía de la voluntad de los pacientes también lo garantiza. Sin embargo, es importante resaltar que aun cuando se refiere a un derecho importantísimo en la sociedad, existen determinados elementos o supuestos que lo limitan (González Fernández et al., 2012; Pérez Dávila, 2019). 
Capacidad de los pacientes: se reconoce la capacidad de los pacientes como una limitante de este consentimiento, porque el derecho de autonomía de la voluntad se determina como un derecho personalísimo. En este sentido, en principio, cuando el propio paciente no cuenta con esa capacidad, se observa un tanto vulnerado ese derecho. Sin embargo, para darle solución a este problema se acepta el consentimiento por representación. En principio, se presume que el representante legal del paciente quiere su bien y no actuará de mala fe.

Renuncia expresa del paciente: el paciente como un ser totalmente autónomo y libre para decidir, puede decidir no ser informado sobre su diagnóstico médico. Esa renuncia debe realizarse previamente y de forma expresa por el enfermo. Al respecto, se toma también como solución el consentimiento sustituto, pues en estos casos debe existir algún familiar que el propio paciente haya decidido, o cualquier otro, para que sea informado y consienta la actuación médica (Zárate Cárdenas, 2003).

Revocación o modificación expresa del paciente: la revocación o modificación del paciente debe realizarse siempre por escrito, luego de haberse otorgado el consentimiento y antes de comenzar a efectuarse el correspondiente tratamiento médico.

Urgencia del tratamiento médico: se refiere a que no se hará uso del consentimiento informado cuando se encuentre en peligro inminente la vida del paciente. En tales casos se observan dos presupuestos: el primero se refiere a los casos de riesgo inmediato grave para la integridad física o psíquica del paciente, donde el consentimiento puede obviarse; el segundo presupuesto se refiere a los casos de riesgo grave para la salud pública o la vida del paciente, en los cuales se podrá utilizar el consentimiento por representación (Cañete et al., 2012).

Hasta aquí se aprecia que el consentimiento informado en la relación médicopaciente se fundamenta doctrinalmente en un conjunto de aspectos necesarios para su mejor comprensión, legalización y aplicabilidad. Tales elementos son expresión concreta de los atributos con los que se distingue de otras instituciones médicojurídicas. Así, el estudio de sus requisitos y límites para su aplicación posibilita que se entienda su desarrollo en la práctica médica, estableciendo sus dos fases: informativa y de decisión.

\section{LA AUTONOMÍA DEL PACIENTE. CONCEPTO, PRINCIPIOS Y FUNDAMENTOS TEÓRICOS}

Es necesario la sociedad entienda la importancia que tiene la aplicabilidad del consentimiento informado en las relaciones entre los médicos y sus pacientes. Ello se debe a que como institución se fundamenta y legaliza a través del principio de autonomía de la voluntad exaltado por la bioética médica. Este principio se desarrolla en función de garantizarle a la sociedad su derecho de decidir con autonomía sobre su estado de salud y tratamientos a realizar. Son estas las razones que propician el 
análisis de tres elementos que se interrelacionan con esta institución: la ética médica, el principio de autonomía de la voluntad y el derecho a la información sanitaria.

Los médicos actuaban tradicionalmente con todo su saber, prestigio y humanidad a favor de los pacientes. Estos tomaban una posición pasiva y se limitaban a cumplir las exigencias del primero, porque tenían un mismo fin: acabar con la enfermedad, el dolor y evitar la muerte. Actualmente se pasa de una relación paternalista y vertical, hacia una visión plural y participativa de los pacientes. Esta afirmación se fundamenta en la evidencia que muestra un aumento en las exigencias de los pacientes, o sus familiares, de responsabilidad a los médicos, frente a un resultado adverso (Perin, 2020; Rodríguez Vázquez, 2012, 2017, p. 35).

También se encuentra la necesidad urgente de proporcionar una protección adecuada a los enfermos, por considerarse el lado más débil de la relación. Como consecuencia de lo planteado, se comprende que los profesionales médicos deben admitir el respeto de la dignidad humana de sus pacientes y no le pueden imponer tratamientos médicos no autorizados por él. Estas ideas se fundamentan precisamente en la bioética, que se conoce como la ciencia que estudia los principios éticos que se relacionan con la actuación adecuada del ser humano sobre la vida. Pero de manera más específica, se corresponden con la ética médica, constituida en los principios que rigen la conducta de los médicos (Alonso Ortega y Tellado, 2004; Legarda Aragón y Henao Vallejo, 2011).

El médico por su propia profesión se encuentra en la obligación de que sus actos se fundamenten en hacer el bien a sus pacientes (principio de beneficencia). En el presente, el paciente hace valer cada día más su autonomía, y la sociedad debe encontrar una respuesta y una interpretación a la calidad de estas nuevas relaciones para poder cumplir con éxito el principio de la justicia social. Es precisamente la bioética médica la que pretende cumplir el encargo de equilibrar estos tres principios (beneficencia, autonomía y justicia social) que no son complementarios sino, la mayoría de las veces, conflictivos (Chiu Navarro et al., 1999).

La bioética juzga los actos médicos con base en cuatro principios fundamentales: justicia, no maleficencia, beneficencia y autonomía. El primero advierte la disminución de situaciones de desigualdad ideológica, social, cultural, económica, entre otras, que puedan existir entre los pacientes. Además, representa el desempeño de los médicos sin que exista una distinción entre los enfermos que atienden, ni cambie el trato profesional por las características divergentes que puedan tener. El principio de no maleficencia se refiere a la obligación que debe tener el profesional de la medicina de abstenerse intencionadamente de realizar acciones que puedan causar daños innecesarios a sus pacientes. Se emplea el término innecesario, porque en la práctica médica, en varias ocasiones, se deben realizar procederes dañinos, de medio a fin, para obtener un bien mayor. Por ejemplo, existen casos como la patología de la diabetes, en los que 
es necesario, para salvar la vida de los que la padecen, amputarle el pie o la pierna. Así, en la enfermedad de pie diabético, cuando no se trata a tiempo y no se toma el riesgo menor, se puede obtener un daño mayor (la muerte) (Risco Turiño et al., 2016).

La no maleficencia se relaciona mucho con el principio de beneficencia. Este alude al deber del médico de actuar siempre en beneficio de los enfermos atendidos por él, promover sus legítimos intereses y suprimir sus prejuicios. La aplicabilidad de este principio supone la promoción del mejor interés de los pacientes, sin tener en cuenta su opinión. Ello se debe a que se entiende que el médico posee conocimientos de los que el paciente carece y, por tanto, aquel sabe y decide lo más conveniente para este. Claramente se puede apreciar cómo estos dos principios de la bioética médica vulneran el derecho de autonomía de los pacientes. Lo cual se debe a que se entiende a los médicos como aquellas personas que tienen los conocimientos necesarios para salvar vidas. Y que, por su ética profesional, se espera que los mismos no provoquen daños a sus pacientes, pues se cree que van siempre a actuar en beneficio de estos. Esta razón que permite comprender como innecesaria cualquier pregunta que se les pueda realizar a los enfermos ya que podrían confiar ciegamente en los médicos.

El principio de autonomía de la voluntad debería ser el que más se respete y garantice en toda sociedad, precisamente porque por su contenido se fundamenta en el principal valor del ser humano. En este tenor, como afirmaron Alonso Ortega y Tellado (2004):

El principio de autonomía en bioética, es el principio supremo ante el cual deben ceder los demás principios. Ello es así, por cuanto la dignidad de la persona humana es el valor que se halla en la cúspide de la jerarquía axiológica (jerarquía de valores). (p. 1)

Este principio es un desplazamiento conceptual, o la materialización misma del derecho a la libertad de elección y el respeto a la dignidad humana como principios universales. En el ámbito de la medicina es el presupuesto jurídico que les permite a los pacientes determinar libremente sobre su estado de salud. En este sentido, es un derecho que le garantiza a un sujeto la capacidad de poder determinarse como un ser totalmente autónomo y libre sobre el tratamiento correspondiente a su patología. La autonomía de la voluntad como derecho se sustenta en el reconocimiento de la libertad y la dignidad humana consagrados en la Declaración Universal de los Derechos del Hombre (1948); (Legarda Aragón y Henao Vallejo, 2011; Sangüesa Cabezudo, 2012).

Este principio presupone el derecho a la información y el respeto a las decisiones tomadas por los pacientes. De esta manera, permite determinarlo como elemento necesario para el otorgamiento del consentimiento informado a través de la voluntad autónoma de los pacientes, la garantía y el respeto a su derecho a la información. Ello supone que se le pueda explicar a los mismos todo cuanto puedan conocer sobre su estado de salud, tratamiento correspondiente, posibles riesgos y beneficios al respecto. 
También comprende este derecho la decisión del enfermo de no ser informado sobre determinada enfermedad.

La información se debe ofrecer de manera verbal y se debe dejar constancia escrita de ese acto. En esa tesitura, la autora Sangüesa Cabezudo (2012) expone, en concordancia con la Ley 41 (2002) española de Sanidad, que debe constar en la historia clínica. Sobre esto, se estima conveniente que la información ofrecida por el médico a sus pacientes se anexe al documento del consentimiento informado como documento que asegura la materialidad del acto. Por otro lado, al ser informado el paciente, también se puede informar a sus familiares, pero el propio titular de este derecho puede decidir no informarle a la familia sobre la realidad sanitaria a resolver. En este sentido, la correcta aplicabilidad del consentimiento informado en las relaciones médico-pacientes dependen necesariamente de cómo los médicos entienden a los enfermos en dicha relación. Deben comprenderlos como seres humanos que pueden decidir sobre determinadas cuestiones relacionadas con su vida. Esa necesidad se encuentra en la argumentación de los principios de la bioética médica, específicamente en el de autonomía de la voluntad.

\section{EL CONSENTIMIENTO INFORMADO EN CUBA. MARCO NORMATIVO}

El consentimiento informado no encuentra en nuestro país un efectivo anclaje jurídico, en correspondencia con las tendencias actuales donde la autonomía de la voluntad suele ser un derecho internacionalmente reconocido. Sin embargo, esto no significa que haya sido totalmente preterido en nuestro ordenamiento jurídico. En las siguientes líneas se presenta un estudio de esta institución y sus principales coordenadas en las distintas legislaciones internas, desde el nivel superior en la escala jerárquica hasta el más local en la provincia de Guantánamo, en el que se ilustran los logros y desafíos en torno al reconocimiento legal de la institución del consentimiento informado.

Se considera a la autonomía de la voluntad de las personas, como un derecho constitucional, por lo que la sociedad cubana puede hacer uso de la libre decisión y la autodeterminación. El preámbulo de la Constitución de la República de Cuba (2019) se manifiesta al respecto, al acogerse a las palabras del apóstol nacional José Martí (1891), cuando expresó "Yo quiero que la Ley primera de la República sea el culto de los cubanos a la dignidad plena del hombre" (Constitución de la República de Cuba, 2019, p. 2). Dignidad que en palabras de Fernández Viciedo (2010) se manifiesta en la autodeterminación consciente y responsable de la propia vida. Por esta razón, se garantiza este derecho para la sociedad, desde la cúspide de la pirámide jerárquica.

La capacidad jurídica de los pacientes es importante en la aplicabilidad del consentimiento informado, y la Ley 59 (1987) del Código Civil es la que regula este tema en los artículos del 29 al 32. Allí se manifiesta que los mayores de dieciocho 
años de edad cumplidos y los menores que han contraído matrimonio tienen la plena capacidad para la toma de decisiones. Esta ley exterioriza, además, que ostentan su capacidad restringida los que han cumplido diez años de edad y puedan tener acceso al estipendio que se les ha asignado; los que han arribado a la edad laboral (diecisiete años de edad) y disponen de la retribución por su trabajo; los que padecen de enfermedad o retraso mental que no los priva completamente de discernimiento; y los que padecen de algún impedimento físico que les impide expresar su voluntad inequívocamente (Ley 59,1987).

Asimismo, dicha ley refrenda en el artículo 31 que no pueden realizar ningún acto jurídico y, por lo tanto, se les exime de la posibilidad de la toma de decisiones propias, a los menores de diez años de edad y a los mayores de edad que han sido declarados judicialmente incapaces. En el artículo 586, en relación al artículo 223, de la Ley 7 (1977) de Procedimiento Administrativo, Laboral y Económico (Lpcale), se realiza dicha declaración judicial a través de un proceso ordinario de expediente de incapacidad, en el que el tribunal competente determina quién será el representante legal de ese mayor de edad incapaz. Cuando se trate de la incapacidad o capacidad restringida de los pacientes, se emplea el consentimiento por representación. En los casos en los que la persona incapaz sea menor de edad, ese consentimiento lo ofrecen sus representantes legales que serían sus padres o tutores. Por otro lado, cuando el incapaz sea mayor de edad, la representación a tales efectos lo realizan, en ese orden, el cónyuge; los hijos mayores de edad, incluyendo los adoptivos; los padres, incluyendo los adoptantes; los hermanos mayores de edad; los abuelos y nietos mayores de edad; y los parientes consanguíneos en línea colateral hasta el tercer grado o los parientes afines hasta el segundo grado (Valenzuela y González, 2010).

Al ser el consentimiento informado una institución aplicada en el marco de las relaciones médico-pacientes, y ser la convergencia de dos disciplinas: la jurídica y la médica, es necesario examinar su regulación legal en el ámbito médico. En este sentido, en los artículos 18 y 19 de la Ley 41 (1983) de Salud Pública reconoce esta institución de forma tácita y no utiliza el término de consentimiento informado en ninguno de los tres artículos en los que se aproxima a esta institución. Solo manifiesta que aquellos procederes quirúrgicos o de diagnósticos, que impliquen riesgos para la persona, deben efectuarse con la aprobación de los pacientes, familiares o tutores legales cuando se trate de menores de edad o de personas incapaces mentalmente. Por otro lado, el artículo 35 refleja la presencia aun de ese carácter paternalista, pues regula que la decisión de realizar algún proceder médico en todo el país le corresponde al personal sanitario facultado para ello. En tales casos, solo ampara el derecho de información que tienen los pacientes.

La próxima legislación de carácter general que le sigue a la Ley de Salud Pública piramidalmente, es la Resolución 1 (2007) del Reglamento General de los Hospitales. 
Esta regula como obligación de todos los trabajadores sanitarios que se le informe adecuadamente a todo aquel que solicite sus servicios, guiándose siempre por los principios de la ética médica socialista. Principalmente, como obligación de los médicos en particular, ampara el deber de informarles a los pacientes y familiares sobre los riesgos del proceder a realizar, y obtener su consentimiento al respecto, basándose en los principios éticos ya mencionados.

Dicha resolución utiliza todo el capítulo xv, artículo 162-f, para referirse al derecho de información de los pacientes y exponer el otorgamiento por escrito de la misma, cuando se trate de investigaciones que generen riesgos para el paciente. En tal apartado, artículo 133, se reconoce este derecho como un elemento importante para lograr la posterior decisión de los pacientes o familiares y mejorar la calidad de la atención médica. Asimismo, en los artículos 134 y 137, ampara a esta información sanitaria como una obligación de los médicos, quienes deben ofrecerla en los términos adecuados, comprensibles y suficientes para que los pacientes o familiares puedan otorgar o no su consentimiento.

Este reglamento constituye la base de la Comisión de Ética Médica que debe existir en cada uno de los centros hospitalarios del país. En este sentido, la Resolución 1 (2007) en los artículos 83 y 84 ampara la necesidad de la creación de la misma por la dirección de los hospitales, quienes determinan su estructura, funciones, atribuciones y, además, garantiza la correcta aplicación de los valores éticos en salud. Así como la identificación y solución de los posibles problemas que se puedan dar en las instituciones sanitarias al respecto.

El artículo 119 de la citada resolución también refrenda que la historia clínica de cada paciente es el documento que recoge toda la información sobre la atención médica ofrecida al mismo. Por tal motivo, consideramos que debe ser donde se anexe el documento de consentimiento informado realizado por escrito. Por otro lado, en caso de ser este de manera verbal, se debe utilizar igualmente un documento en el que se ilustre la consciente decisión del paciente sobre la realización del correspondiente tratamiento.

Sobre esta misma línea jerárquica, la Resolución 135 (2008) del Reglamento General de los Policlínicos es la siguiente legislación analizada. Esta refleja un vacío legislativo respecto al consentimiento informado, pues regula cuestiones administrativas, de calidad y servicios de los policlínicos. No obstante, reconoce en su capítulo xi "De los derechos y deberes de los pacientes y sus familiares", el acceso de estos a la información sanitaria como un derecho (igualmente lo garantiza en su artículo 91), a que se le respete su pudor y se les trate con amabilidad y respeto (artículo 48). Además, regula como base de la calidad de los servicios sanitarios, los principios y los valores de la ética médica socialista en los artículos 7 y 18-b. 
A pesar de la escasa regulación del consentimiento informado, analizado anteriormente, se evidencia que en Cuba se reconoce esta institución para algunos procederes sanitarios importantes en los que se generan riesgos para los pacientes. Tal es el caso de la terminación voluntaria de los embarazos (TVE). La Resolución 24 (2013) en el artículo 3-b contempla entre los requisitos necesarios para la realización de este proceder, el consentimiento informado expresamente por escrito de la embarazada o su representante legal cuando proceda.

$\mathrm{Al}$ adentrarnos en disposiciones legales más específicas sobre la materia investigada, se analizan resoluciones del Hospital General Docente de Guantánamo. Tal análisis legislativo se fundamenta en el estudio del reglamento funcional interno (2019) y el reglamento disciplinario interno (2014). de la propia entidad. El primero, es la norma aprobada por la administración de la entidad en función de darle respuesta al Reglamento General de los Hospitales (2007). Por tal motivo, se afirma que en el reglamento funcional interno (2019) del Hospital General Docente de Guantánamo, se reconoce el consentimiento informado y el derecho de información de los pacientes, de igual forma que en la Resolución 1 (2007), previamente estudiada.

Por otro lado, el reglamento disciplinario interno (2014) es la norma que regula las indisciplinas laborales cometidas por el médico en su actuar y sus correspondientes medidas aplicadas. Por ello, es importante su análisis, pues consideramos que se debe reconocer dentro de las violaciones de los principios éticos en la profesión sanitaria, que generan gran responsabilidad médica, la no aplicación del consentimiento informado.

Tal reglamento en su artículo 4-c les prohíbe a todos los trabajadores de la entidad no respetar de la dignidad de las personas y realizar actos contrarios a la ética y a la moral de las mismas. De esta manera, en el artículo 65-d determina como una indisciplina de suma gravedad, la realización de esta prohibición, cuando genere daños importantes a las personas, para la cual se determina como medida disciplinaria, la separación definitiva de la actividad. Además de ello, en artículo 7-b y 7-e reconoce como obligación de los médicos, el derecho de información sanitaria de los pacientes y la obtención del consentimiento informado de los mismos para todos los procederes en los que se generan riesgos. Otro elemento importante y novedoso que acoge esta legislación en su artículo 12, es el reconocimiento de que las enfermeras no constituyen sujetos directos, o primarios del proceso de consentimiento informado.

Todo este análisis legislativo permite corroborar la idea que ya se tenía al respecto. Aun cuando se reconoce constitucionalmente el derecho de autodeterminación, las legislaciones analizadas no desarrollan de forma efectiva la institución del consentimiento informado. Se puede reafirmar que existe un vacío normativo sobre esta institución, tanto en las disposiciones jurídicas de aplicación nacional como en las emitidas en el centro de salud objeto de estudio. No obstante, el personal de 
los servicios sanitarios ha planteado que en la provincia se utilizan modelos de aplicación de este consentimiento para determinados procederes. Por esta razón, se estima importante realizar un estudio de las diferentes proformas de consentimiento informado utilizados en la práctica médica cubana.

\section{PROPUESTA PARA SU ADECUADA CONFIGURACIÓN NORMATIVA}

Debido a las diferentes deficiencias demostradas en el desarrollo de esta investigación, es necesaria la actualización o correcta regulación del consentimiento informado en Cuba (Risco Turiño et al., 2016). Por tal motivo se realiza la propuesta normativa necesaria para la adecuada regulación legal de tal institución médicojurídica y sus elementos teóricos doctrinales, en aras de poder lograr su correcta aplicabilidad en las relaciones médico-pacientes.

La mayor deficiencia encontrada es la referida a la insuficiencia legislativa existente tanto en la Ley de Salud Pública (1983), como en cada una de las resoluciones específicas que fueron analizadas. Consecuentemente, se propone que el Ministerio de Salud Pública emita una resolución en la que se regule adecuadamente la institución del consentimiento informado, el derecho a la información sanitaria y el principio de autonomía de la voluntad de los pacientes. Para la propuesta de esta norma, se analiza la Resolución 223 (2018) del Ministerio de Justicia (Minjus) en la que se determina la metodología para la redacción de las resoluciones.

Estas resoluciones se componen por tres partes: i) la expositiva, que se divide en párrafos separados denominados por cuantos, en los que se consignan los fundamentos de hecho y de derecho para dictarla; ii) la parte dispositiva que constituye el objeto de regulación, compuesta por los artículos o apartados, en su caso; y iii) la parte final, conformada por las disposiciones especiales, transitorias y finales, cuando proceda. En estas líneas se hace una alusión breve a la parte expositiva, y con mayor énfasis en la dispositiva, precisamente porque es la que se encarga de resolver el asunto y concretar los elementos a regular jurídicamente. El encabezamiento no constituye un eje importante en este análisis dispositivo. En él se expresa el número consecutivo de las resoluciones emitidas en un año, cuestión que es decisión propia del Ministerio de Salud (quien la emite) según su protocolo de resoluciones.

La parte expositiva se compone por los por cuanto, que disponen los fundamentos de derecho y de hecho. En los primeros de ellos (de derecho), se establecen las normas que facultan a la Dirección del Ministerio Salud Pública en Cuba para emitir tal disposición. Además, se concretan cada una de las cuestiones que legalmente reconocen y fundamentan la realización de tal acción jurídica. Los segundos (de hecho), establecen claramente los hechos en los que se basa la disposición normativa. En el último apartado de esta parte expositiva, denominado por tanto, se consigna la facultad del Ministerio de Salud Pública, para resolver, y se continúa con la parte dispositiva. 
Esta parte comienza con la palabra resuelvo en el medio, y posteriormente enumerados de manera ascendente y con letra se enuncian los apartados que regulan directamente las cuestiones a resolver. En el primer resuelvo se dispone la aprobación y puesta en vigor de la resolución, expresando su título, que se considera adecuado: "Sobre el consentimiento informado y la autonomía de los pacientes". El segundo resuelvo se debe referir a los derechos y deberes de los pacientes y familiares en la relación médico-paciente. Aquí se debe regular el derecho de los mismos a su autonomía, sobre todo proceder sanitario a efectuarse, a que los médicos le reconozcan su derecho a recibir información de manera clara y precisa sobre el diagnóstico de su enfermedad, y que le respeten su libre decisión de aceptar o no el tratamiento correspondiente. Asimismo, debe disponerse el deber de los pacientes de facilitar a los médicos que los atienden, todos los datos sobre su estado físico o su salud de manera leal y verdadera.

El siguiente resuelvo debe consignar elementos de la información sanitaria: los titulares de esta (quienes deben ser informados) y cuándo debe informársele a los familiares o tutores sobre su contenido, calidad, cantidad y la limitante de su uso, cuando exista una necesidad terapéutica ${ }^{2}$. En este sentido, el profesional debe dejar constancia (en la historia clínica) de tal necesidad.

En el cuarto resuelvo debe reconocerse el consentimiento informado. El mismo debe estar compuesto por el concepto de esta institución y la necesidad de que en la manifestación de voluntad de los pacientes se encuentren ausentes: la amenaza, la manipulación y el engaño. Además, se necesita establecer aquí la aplicación de ese consentimiento en todos los procederes médicos realizados, explicándo se siempre que, por ley general, será de manera verbal y en los casos en los que se genere riesgos para los enfermos se realizará por escrito.

En ambos supuestos se deben insertar los documentos ${ }^{3}$ a la historia clínica de los pacientes. De esta manera, cuando el consentimiento informado se realice de forma verbal, el médico debe anexar a la historia clínica un documento en el que se exprese que se aplicó esta institución de esa forma, y que el paciente estuvo de acuerdo o no con el mismo, además, debe estar firmado por el médico y el enfermo. En el caso del consentimiento realizado por escrito, se debe insertar en tal historia el modelo de consentimiento informado que se llevó a cabo y que fue debidamente

2 La necesidad terapéutica es la facultad del médico para actuar profesionalmente sin informar antes al paciente, cuando por razones objetivas el conocimiento de su propia situación pueda perjudicar su salud de manera grave y en tales casos se le debe informar a las personas vinculadas al paciente por razones familiares o de hecho.

3 Estos documentos se refieren a que en los casos de consentimiento informado se realice de forma verbal, el médico debe anexar a la historia clínica de sus pacientes un documento en el que se exprese que se aplicó esta institución de forma verbal, y que el paciente estuvo de acuerdo o no con el mismos, aportándose además las firmas del médico y el enfermo. Y en el caso del consentimiento realizado por escrito, se debe insertar en tal historia el modelo de consentimiento informado que se llevó a cabo y que fue debidamente firmado por las partes y los testigos. 
firmado por las partes y los testigos. Igualmente, se debe determinar con claridad en este resuelvo los sujetos que intervienen en el proceso de aplicación de esta institución; las personas que están legitimados para otorgar el consentimiento informado en casos de incapacidad de los pacientes; los requisitos necesarios para poder aplicarlo, y las excepciones en los que no se puede emplear.

\section{CONCLUSIONES}

El consentimiento informado es una institución que se manifiesta en la relación médico-paciente, desarrollada en un proceso en el que el médico debe informarles a sus pacientes, con los términos comprensible, sobre el diagnóstico de su enfermedad, los posibles tratamientos correspondientes, y los riesgos y beneficios que se pudieran obtener. De la misma manera, el médico debe esperar que los mismos decidan libre y voluntariamente respecto a la realización o no de determinado proceder médico.

La aplicación del consentimiento informado constituye una expresión del reconocimiento social y jurídico proporcionado al principio bioético de autonomía de la voluntad, como un derecho constitucionalmente reconocido a la libre autodeterminación de la persona y también del derecho a la información sanitaria. Para su empleo adecuado se deben tener en cuenta: sus elementos como institución jurídica (sujetos, objeto y forma), sus características, fases, requisitos y límites.

La práctica médica cubana se caracteriza por hacer una regulación insuficiente del consentimiento informado, pues no lo reconoce apropiadamente en la Ley de Salud Pública (1983) y, consecuentemente, las demás normas de menor jerarquía jurídica tampoco lo hacen. Además, en la sociedad, tanto profesionales médicos y jurídicos, como pacientes en general, no conocen debidamente la existencia y aplicación de tal consentimiento en las relaciones médico-pacientes. Esta afirmación se fundamenta en la ausencia de proformas de consentimiento informado usados en los diferentes procederes sanitarios que por su naturaleza lo requieren.

\section{REFERENCIAS}

Agón López, J. G. (2016). Consentimiento informado y responsabilidad médica Itesis doctoral, Universidad de Salamancal. Gredos. Gestión del Repositorio Documental de la Universidad de Salamanca. https://gredos.usal.es/handle/10366/135700

Alfaro Carballido, L. D., y García Rupaya, C. R. (2011). Percepción del proceso de consentimiento informado en pacientes de la Clínica Estomatológica Central de la Universidad Peruana Cayetano Heredia. Revista Estomatológica Herediana, 21(1), 5-12. https://doi.org/10.20453/reh.v21i1.1720

Alonso Ortega, A., y Tellado, M. (2004). Sobre la libertad en la elección del tratamiento médico. Persona, 25. http://www.revistapersona.com.ar/Persona25/25Alonso.htm

Arrue Caraballo, R., y Azaharez Leal, G. (2012). Del error médico a la responsabilidad penal, cuestión esencial del tema probando. Una mirada crítica al procedimiento establecido en Cuba. En A. 
Mantecón y E. Moya (eds.). Abogacía y Derecho: Gestión de conflictos jurídicos. Vol I (pp. 41-63). Ediciones ONBC.

Asamblea Nacional de la República de Cuba. (1977, 19 de agosto). Ley de Procedimiento Civil, Administrativo, Laboral y Económico con las modificaciones del Decreto-Ley 241 de 2006. Gaceta oficial de la República de Cuba. https://bit.ly/3r1S6Xt

Asamblea General de las Naciones Unidas. (1948, 4 de noviembre). Declaración Universal de los Derechos del Hombre. https://www.humanium.org/es/derechos-humanos-1948/

Asociación Médica Mundial (1964). Declaración de Helsinki. Declaración de Helsinki de la AMM-principios éticos para las investigaciones médicas en seres humanos. https:/www.wma.net/es/policies-post/ declaracion-de-helsinki-de-la-amm-principios-eticos-para-las-investigaciones-medicas-en-sereshumanos/

Bandrés, F., Caballero, M., Delgado, S., González, M., Herreros, B., Pinto, P., Zarco, C. (2010). El Consentimiento Informado. Comité de Bioética y Derecho Sanitario de ASISA-LAVINIA.

Bernar Borda, A. (2000). Valoración moral del consentimiento informado como expresión de la relación médicopaciente [tesis doctoral, Pontificia Universidad de la Santa Cruz, Romal. Gestión del Repositorio Documental de la Universidad de la Santa Cruz, Roma https://www.bioeticacs.org/iceb/ investigacion/tesis_doctoral_CI.pdf

Cadenas Osuna, D. (2018). El consentimiento informado y la responsabilidad médica [tesis doctoral, Universidad Pablo de Olavide]. Repositorio Institucional UPO. https://rio.upo.es/xmlui/handle/10433/6279.

Cañete, R., Guilhem, D., y Brito, K. (2012). Consentimiento informado: algunas consideraciones actuales. Acta Bioethica, 18(1), 121-127. http://dx.doi.org/10.4067/S1726-569X2012000100011

Carta de los Derechos del Paciente (1973) https://www.codem.es/Adjuntos/CODEM/Documentos/ Informaciones/Publico/f044efef-58a7-40e4-bb7b-91605df12553/B4694157-ADF7-4DD2A221-229B18C518C7/18fb7dc6-1d99-4c69-a0f9-7d09b99f12de/18fb7dc6-1d99-4c69-a0f97d09b99f1 2de.pdf

Casa Madrid Mata, O. (2004). Origen del consentimiento informado bajo información. Revista Conamed, 9(3), 14-22. https://www.medigraphic.com/cgi-bin/new/resumen.cgi?IDARTICULO=79870

Constitución italiana (1947). http://www.derecho.uba.ar/publicaciones/lye/revistas/34/la-constitucionitaliana-de-1947.pdf

Chepe Ramos, E. E. (2010). Acto médico y el consentimiento informado.Fliphtme5. https://fliphtml5.com/ nwzd/mkxn/basic.

Chiu Navarro, V., Colás Costa, M., Alonso Pardo, M. E., y Cossío Figueroa, N. (1999). Algunas reflexiones de la bioética en las ciencias médicas. Educación Médica Superior, 13(1). http://scielo.sld.cu/scielo. php?script=sci_arttextEpid=S0864-21411999000100002Elng=esEnrm=isoEtlng=es

Código Civil de la República de Cuba. (1987 de 16 de julio) Ley 59 de 1987. Pérez Gallardo, L. B. (2011). Editorial de Ciencias Sociales.

Convenio de Oviedo (1997). Revista Anales de la Facultad de Medicina Universidad Nacional Mayor de San Marco, año/vol. 62, número 003. http://www.bioeticanet.info/documentos/Oviedo 1997.pdf

Constitución de España. Cortes en sesiones plenarias del Congreso de los Diputados y del Senado. (31 de octubre de 1978). https://www.boe.es/legislacion/documentos/ConstitucionCASTELLANO.pdf 
Comisión Nacional de Bioética. (1947, 20 de agosto). Código de Núremberg. Repositorio institucional Conbioética-Mexico. http://www.conbioetica-mexico.salud.gob.mx/descargas/pdf/normatividad/ normatinternacional/2.INTL._Cod_Nuremberg.pdf

Constitución de la República de Cuba. (2019, 10 de abril). Cubadebate.cu http://www.cubadebate.cu/ noticias/2019/04/09/descargue-la-constitucion-de-la-republica-de-cuba-pdf/

Cortes Generales de España (1986, 25 de abril). Ley General de Sanidad. Boletín Oficial del Estado n. ${ }^{\circ}$ 102. https://www.boe.es/boe/dias/1986/04/29/pdfs/A15207-15224.pdf

Danel Ruas, O. O. (2020, 3 de enero). Logros de la salud cubana. [Conferencia], Universidad de Ciencias Médicas de La Habana https://www.researchgate.net/publication/338400955_logros_de_la_salud_ publica_cubana_.

Director Unidad Presupuestada de Salud Hospital Clínico Quirúrgico-Ginecobstétrico "Dr. Antonio Agostinho Neto" Provincia Guantánamo (2014, 5, octubre). Resolución No. 67 Reglamento disciplinario interno. Protocolo de Resoluciones del Hospital en la oficina del Asesor Jurídico.

Directora Unidad Presupuestada de Salud Hospital Clínico Quirúrgico-Ginecobstétrico "Dr. Antonio Agostinho Neto" Provincia Guantánamo (2019, 12, diciembre). Resolución No. 72 Reglamento funcional interno. Protocolo de Resoluciones del Hospital en la oficina del Asesor Jurídico.

Fernández Viciedo, Y. (2010). ¿Es posible fundamentar la eutanasia voluntaria en Cuba? Un acercamiento jurídico al fenómeno. Contribuciones a las Ciencias Sociales, 1-11. www.eumed.net/rev/cccss/09/yfv.htm

Gómez Rivero, M. del C. (2003). La responsabilidad penal del médico. Tirant lo Blanch.

González Fernández, E., Pardo Vitorero, R. y Lombera Torre, Á. (2012). Consentimiento informado y práctica profesional. Nuberos Científica, 1(7), 15-21. http://nc.enfermeriacantabria.com/index.php/ nc/article/view/68/65

González López, S. L., Cabrera Machado, C. A., Cortiza Orbe, G., Quintero Delgado, Z., Fonseca Romero, B. E. y Ponce Rodríguez, Y. (2009). Relación médico paciente y consentimiento informado en cirugía pediátrica: teoría y realidad en la práctica local. MediSur, 7(4), 29-36. https://www.redalyc. org/articulo.oa?id $=180014084006$

Legarda Aragón, I. I., y Henao Vallejo, L. F. (2011). El consentimiento informado en la relación médico paciente en la ciudad de Medellín: análisis jurisprudencial. Revista Ces Derecho, 2(2), 134-153. https:// revistas.ces.edu.co/index.php/derecho/article/view/1991

Jefatura del Estado. (2002, 14 de noviembre) Ley 41 de 2002, básica reguladora de la autonomía del paciente $y$ de derechos y obligaciones en materia de información y documentación clínica. Boletín Oficial del Estado. https://www.boe.es/buscar/pdf/2002/BOE-A-2002-22188-consolidado.pdf

Lifshitz, A. (2015). El futuro de la relación médico-paciente. Gaceta Médica de México, (151), 437. https:// www.medigraphic.com/cgi-bin/new/resumen.cgi?IDARTICULO $=60906$

Loring Martínez de Irujo, P. (2013). Consentimiento Informado. Doctrina del Tribunal Supremo. Universidad Internacional de Andalucía. https://dspace.unia.es/bitstream/handle/10334/2581/0504_Loring. pdf?sequence $=1$.

Lugones Botell, M., Pichs García, L. A., y García Hernández, M. (2005). Consentimiento Informado. Rev Cubana Med Gen Integr, 21(5-6), 1-7. http://scielo.sld.cu/pdf/mgi/v2 ln5-6/mgi195-605.pdf.

Martí Pérez, J.J. (1981). "Discurso Pronunciado en el Liceo Cubano", en: Obras completas. (vol. 4). Editorial Nacional de Cuba. (1963-1973). 
Mellado, J. (2016). Autonomía, consentimiento y responsabilidad. Parte 1: limitaciones del principio de autonomía como fundamento del consentimiento informado. Radiología, 58(5), 343-351. https:// doi.org/http://dx.doi.org/10.1016/j.rx.2016.06.009

Menacho Ángeles, G. L. (2018). Consentimiento Informado, parte fundamental en la Historia Clínica. Exégesis. Revista de la Escuela de Posgrado, 57-59. http://revistas.uigv.edu.pe/index.php/exegesis/ article/view/629

Ministerio de Salud Pública de la República de Cuba. (1983, 13 de julio). Ley No. 41 Ley de la Salud Pública. Gaceta Oficial de la República de Cuba, Ordinaria, Nº 61, de 15 de agosto de 1983.

Ministerio de Salud Pública de la República de Cuba. (1988, 4 de febrero). Decreto No 139 Reglamento de la Ley de la Salud Pública. Gaceta Oficial de la República de Cuba, Ordinaria, No 12, de 22 de febrero de 1988.

Ministerio de Salud Pública de Cuba. (2007, 9 de enero). Resolución Ministerial 1 de 2007. Reglamento General de Hospitales.

Ministerio de Salud Pública de Cuba. (2008, 17 de junio). Resolución Ministerial 135 de 2008. Reglamento General de Policlínico.

Ministerio de Justicia de la República de Cuba. (2018, 4 de noviembre). Resolución No. 223 «Metodología para la elaboración de resoluciones e instrucciones». Gaceta Oficial No. 76 Extraordinaria de 5 de diciembre de 2018 (GOC-2018-833-EXT76).

Ministerio de Salud Púbica de la República de Cuba. (2013, 23, Marzo). Resolución 24 sobre terminación voluntaria de los embarazos (TVE).

Morales Ojeda, R., Mas Bermejo, P., Castell-Florit Serrate, P., Arocha Mariño, C., Valdivia Onega, N. C., Druyet Castillo, D. y Menéndez Bravo, J. A. (2018). Transformaciones en el sistema de salud en Cuba y estrategias actuales para su consolidación y sostenibilidad. Rev Panam Salud Publica, 42(25), 1-8. https://scielosp.org/pdf/rpsp/2018.v42/e25/es

Novoa Reyes, R. H. (2013). Nivel de conocimiento sobre consentimiento informado en la práctica médica en internos de medicina en el Hospital Nacional Dos de Mayo, 2013 [tesis de especialidad, Universidad Nacional Mayor de San Marcos]. Gestión del Repositorio Documental de la Universidad Nacional Mayor de San Marcos https://core.ac.uk/download/pdf/323348859.pdf

Núñez de Villavicencio Porro, F. (2006). Consentimiento educado vs. Consentimiento informado. Revista Cubana de Salud Pública, 32(4). http://scielo.sld.cu/scielo.php?script=sci_arttextEpid=S086434662006000400011 Elng=esEnrm=isoEtlng=es

Ochoa Ulloa, D. P. (2017). Análisis del consentimiento informado por representación en el sistema jurídico ecuatoriano [tesis para obtener el título de abogada, Universidad del Uzuay]. Gestión del Repositorio Documental de la Universidad del Uzuay. http://dspace.uazuay.edu.ec/bitstream/ datos/6897/1/12855.pdf

Patiño Restrepo, J. F. (2012). ¿La tecnología amenaza la relación médico-paciente? Revista Colombiana de Cirugía, (27), 97-98. http://www.scielo.org.co/pdf/rcci/v27n2/v27n2al.pdf.

Peña Machuca, C. R. (2017). El consentimiento informado y su defensa por parte del Estado [Tesis de grado para obtener el título de abogado, Universidad Ricardo Palmal. Gestión del Repositorio Documental de la Universidad Ricardo Palma. https://repositorio.urp.edu.pe/bitstream/handle/urp/1 123 / TESISCarmen\%20Rosa\%20Pe\%C3\%B1a\%20Machuca.pdf?sequence= 1 EisAllowed =y 
Pérez Dávila, B. F. (2017). Implicaiones ético legislativas del consentimiento informado en la redacción del protocolo del aborto terapéutico para la madre gestante [Tesis de maestría, Universidad Católica Santo Toribio]. Repositorio Institucional AC. https://core.ac.uk/download/pdf/160496941.pdf

Pérez Dávila, B. F. (2019). Implicancias bioéticas legislativas del consentimiento informado en la redacción del protocolo de aborto terapéutico. IUS. Revista de Investigación de la Facultad de Derecho, 8(2), 79-95. https://doi.org/10.35383/ius.v1i2.277

Perin, A. (2020). El fenómeno de la medicina defensiva como cuestión político-criminal. ¿Cómo conjugar autonomía y responsabilidad? En C. Romero y A. Perin (eds.). Derecho y medicina defensiva: legitimidad y límites de la intervención penal (pp. 1-23). Editorial Comares, S.L.

Redacción Minsap. (2019, 27 de diciembre). Ministro de Salud expone los principales resultados del sector en el año 2019: "Continuar potenciando a la salud cubana como un referente para el mundo" [Conferencia]. https://salud.msp.gob.cu/ministro-de-salud-expone-los-principales-resultados-del-sector-en-elano-2019-continuar-potenciando-a-la-salud-cubana-como-un-referente-para-el-mundo/

Restrepo, B. D., Cardeño C., C., Duque G., M., y Jaramillo, S. (2012). Del principio de beneficencia al principio de autonomía: aproximación a la evaluación de la competencia mental de los pacientes en el hospital general. Revista Colombiana de Psiquiatría, 41(2), 395-407. http://www.redalyc.org/ articulo.oa?id $=80624462004$

Risco Turiño, C. A. del, Torres Armenteros, A. L., Macías Llanes, M. E., y Risco Veloz, D. del. (2016). El consentimiento informado en las amputaciones mayores de miembros inferiores. Humanidades Médicas, 16(2), 273-284. http://scielo.sld.cu/scielo.php?script=sci_arttextEpid $=$ S1727-81202016000200007.

Rodríguez Vázquez, V. (2012). Responsabilidad penal en el ejercicio de actividades médico-sanitarias. Criterios para delimitar la responsabilidad en supuestos de intervención conjunta de los profesionales sanitarios. Marcial Pons. https://www.marcialpons.es/media/pdf/9788497688925.pdf.

Rodríguez Vázquez, V. (2017). El delito de tratamiento médico arbitrario: una propuesta de lege ferenda. Revista Electrónica de Ciencia Penal y Criminología, 19(3), 1-59. http://criminet.ugr.es/recpc/19/ recpc19-03.pdf.

Sánchez Arrastía, D. y Contreras Olivé, Y. (2014). La relación médico-paciente y su importancia en la práctica médica. Revista Cubana de Medicina Militar, 43(4), 528-533. http://scielo.sld.cu/scielo. php?script $=$ sci_arttextEpid=S0138-65572014000400011

Sangüesa Cabezudo, A. M. (2012, 13 de diciembre). Autonomía del paciente. Consentimiento informado [ponencia]. https ://elderecho.com/autonomia-del-paciente-consentimiento-informado

Santos Echaide, M. de los Á. (2016). Breve recorrido histórico de la relación médico-paciente. Comisión Estatal de Arbitraje Médico de Oaxaca, 3-22. http://www.ceamooax.org.mx/revistas/Sep-Diciembre2016. pdf.

Sanz Rubiales, Á., De Valle Rivero, M. L., Fernández González, M. y Ferreira Alonso, R. (2016). Teoría y práctica del consentimiento informado. Cuadernos de Bioética, xxvII(1), 69-78. http://www.redalyc. org/articulo.oa?id=87545382004

Seuba Torreblanca, J. C., y Ramos González, S. (2003). Derechos y obligaciones en materia de autonomía privada, información y documentación clínica. Presentación de la Ley 41/2002, de 14 de noviembre. InDret, (2), 1-17. https://dialnet.unirioja.es/servlet/articulo?codigo $=369136$ 
Valenzuela, V. T., y González , G. Y. J. (2010). El consentimiento informado y su repercusión en la responsabilidad jurídica civil [Trabajo de curso]. Universidad de La Habana.

Vera Carrasco, O. (2016). El consentimiento informado del paciente en la actividad asistencial médica. Rev Med La Paz, 22(1), 59-68. http://www.scielo.org.bo/pdf/rmcmlp/v22n1/v22n1_a10.pdf

Vila Morales, D. y Pérez Echeverría, R. (2013). Consentimiento informado como institución del derecho médico. En Dadonim Vila Morales. Teoría del derecho médico (pp. 134-183). La Habana: Ciencias Médicas.

Vizcaya Benavides, D. M., Zúñiga Villarreal, F. A., Pérez Cortés, P., y Cobos Aguilar, H. (2014). Conocimiento de los pacientes sobre el consentimiento informado en un hospital general. Revista de la Facultad de Medicina de la UNAM, 57(5), 5-13. http://www.scielo.org.mx/pdf/facmed/v57n5/00261742-FACMED-57-05-00005.pdf

Zárate Cárdenas, E. (2003). Los derechos de los pacientes y el consentimiento informado en Perú. Situa-Revista Semestral de la Facultad de Medicina Humana-Unsaac, xxIII(23), 4-10. https://sisbib.unmsm. edu.pe/bvrevistas/situa/2004_n23/enPDF/a03.pdf 